\title{
Improving Colorectal Cancer Screening in Family Medicine: An Oklahoma Physicians Resource/Research Network (OKPRN) Study
}

\author{
Dewey C. Scheid, MD, MPH, Robert M. Hamm, PhD, \\ Kalyanakrishnan Ramakrishnan, MD, Laine H. McCarthy, MLIS, \\ James W. Mold, MD, MPH, and the Oklaboma Physicians Resource/Research Network
}

Purpose: The purpose of this study was to describe colorectal cancer screening (CRCS) practices across a variety of primary care clinics and identify the methods used by primary care physicians (PCPs) with higher rates of CRCS ("exemplars").

Methods: Physician questionnaires, structured interviews, medical record abstractions, and practice observations were conducted for 48 PCPs in 25 practices within a regional practice-based research network followed by secondary in-depth interviews to further investigate the practices of PCPs in the top quartile of CRCS rates ("exemplars").

Results: We abstracted 3596 medical records (mean of 75 records per PCP). Overall, exemplars had higher CRCS rates (median, $57.2 \%$ vs. $27.6 \% ; P<.001$ ). Patients of exemplars had higher screening rates for fecal occult blood testing (FOBT) and colonoscopy but not for flexible sigmoidoscopy or double-contrast barium enemas. Exemplars adopted few of the system-based innovations proposed by researchers to improve CRCS. Colonoscopy was promoted as the preferred CRCS method. FOBT was recommended for patients who could not afford or did not want colonoscopy. Flexible sigmoidoscopy or barium enemas were rarely recommended. Exemplars used brief CRCS promotion scripts that informally paralleled theory-driven counseling techniques.

Conclusions: Experienced PCPs use brief CRCS promotion scripts including counseling techniques that improve CRCS performance. Future research should be directed toward whether these techniques can be used to create an intervention aimed at PCPs to improve CRCS. (J Am Board Fam Med 2013;26:498-507.)

Keywords: Cancer Screening, Colorectal Cancer, Practice-based Research, Prevention, Primary Health Care

Colorectal cancer screening (CRCS) is recommended by many organizations, including the US Preventive Services Task Force (USPSTF), the American College of Gastroenterology, the American

This article was externally peer reviewed.

Submitted 30 August 2012; revised 3 May 2013; accepted 13 May 2013.

From the Department of Family and Preventive Medicine, University of Oklahoma Health Sciences Center, Oklahoma City, OK.

Funding: Support for this work was provided by the $\mathrm{Na}$ tional Cancer Institute (grant 5R21CA100518).

Conflict of interest: none declared.

Disclaimer: The views and opinions expressed here are those of the authors and do not necessarily reflect the views or opinions of the National Cancer Institute.

Corresponding author: Dewey Scheid, MD, MPH, Department of Family and Preventive Medicine, University of Oklahoma Health Sciences Center, 900 NE 10th Street, Oklahoma City, OK 73104 (E-mail: dewey-scheid@ouhsc.edu).
Cancer Society, the US Multi-Society Task Force on Colorectal Cancer, the American College of Radiology, and the American College of Physicians. ${ }^{1-5}$ Despite near universal endorsement, at least $40 \%$ of eligible individuals have not been adequately screened for colorectal cancer. ${ }^{6}$

A physician's recommendation has been associated with increased receipt of CRCS. ${ }^{7,8}$ Numerous studies regarding methods to improve CRCS rates, such as physician prompts, patient reminder or tracking systems, performance feedback reports, patient education media, decision aids, group education, and one-on-one interactions with health educators or a nurse, have been published. However, relatively few published studies describe what methods have been adopted by primary care physicians (PCPs) and whether they are effective. ${ }^{9}$ A 
Wisconsin survey of 600 PCPs revealed that $58 \%$ had no provider reminder system. ${ }^{10}$ In a national survey of PCPs, Klabunde et a ${ }^{11}$ found that $30 \%$ reported some mechanism to prompt PCPs, $15 \%$ used patient reminders, and only $12 \%$ received reports of CRCS rates for their patient panel. Investigators who analyzed practice performance data from a network of PCPs using a common electronic health record described several strategies used by high-performing practices, including prioritizing performance, progress note templates, wellness visits combined with an opportunistic approach to screening, sigmoidoscopy for self-paying patients, and patient activation through a variety of repeated screening messages. ${ }^{12}$

Less is known about how PCPs discuss CRCS with their patients. ${ }^{13}$ Ling et $\mathrm{al}^{14}$ found a lack of informed decision making during CRCS discussions in 91 audiotaped clinic visits between patients and PCPs in the Veterans Administration Pittsburgh health care system. Direct observation of periodic health examinations found limited use of the 5 A's framework (ask, advise, assess, assist, arrange) during discussions of CRCS. Use of the 5 A's is advocated by the USPSTF for delivery of preventive health services. ${ }^{15}$ Because of these important knowledge gaps, the purpose of this study was to identify PCPs with higher CRCS rates (exemplars) and describe what they did to enhance their delivery of CRCS services.

\section{Methods}

\section{Subjects, Setting, and Recruitment}

This study was conducted within the Oklahoma Physicians Resource/Research Network (OKPRN), a network of clinicians committed to improving primary health care services statewide by sharing resources and conducting practice-based research. At the time of this study, the network included 100 community clinicians (mostly family physicians and physician assistants) in 81 different practices plus 112 faculty and 172 residents in the 3 academic departments of family medicine and 7 community-based residency programs. Seven practices served only $\mathrm{Na}$ tive Americans. Network clinicians cared for more than 325,000 patients, $8 \%$ of whom were Hispanic, $62 \%$ white, $11 \%$ black, $17 \%$ Native American, and $2 \%$ Asian. The University of Oklahoma Health Sciences Center Institutional Review Board approved the study.
A total of 48 PCPs in 25 OKPRN practices were recruited using methods similar to previous OKPRN studies. ${ }^{16-19}$ All PCPs $(\mathrm{n}=72)$ in OKPRN practices that had a concurrently assigned practice enhancement assistant (PEA), also referred to as a practice facilitator, ${ }^{20,21}$ were invited to participate. PEAs are research assistants with master's degrees (eg, in health promotion sciences) who are assigned to act as the functional link between the OKPRN and the Research Division of the Department of Family and Preventive Medicine. Each PEA is assigned to 8 practices, visiting each one every 2 weeks on a predictable schedule; the PEA spends the day assisting the practice with identified objectives and obstacles and meeting with the physicians and key support staff. PEAs conducted practice medical records abstractions and structured interviews of PCPs' methods, similar to those previously reported. ${ }^{16-19}$ PEAs kept detailed unstructured field notes of their observations of CRCS in each practice to confirm and augment answers from structured interviews.

\section{Instruments}

A PCP questionnaire was developed by modifying components of the physician survey from the Direct Observation of Primary Care Study ${ }^{22}$ to describe the organization, patient care activities, and delivery of a wide range of evidence-based preventive services and by using the theoretical frameworks of Cabana et $\mathrm{al}^{23}$ and Woolf, ${ }^{24}$ which organizes barriers to adherence to guidelines according to their relation to physician knowledge, attitudes, and behavior. The questionnaire surveyed 48 PCPs about their knowledge of CRCS recommendations, agreement with CRCS recommendations, beliefs about the effect of CRCS, perceived external barriers to performing CRCS recommendations, and perceived ability to provide effective and efficient CRCS.

Participating PCPs completed a questionnaire containing questions about practice characteristics using the definitions developed for use by primary care practice-based research networks funded by the Agency for Health care Research and Quality and the Practice Environment Checklist developed by the Direct Observation of Primary Care Study. ${ }^{22}$ PCPs and office personnel were interviewed about how they performed CRCS and what tools they used. 
To describe the step-by-step processes of the delivery of CRCS services by PCPs using the framework of the Patient Path Model, ${ }^{25}$ we developed a structured interview that was administered by the PEAs. The interview included more than 100 open-ended, branching questions that identified and captured the details of how CRCS services were delivered and supported before, during, and after a patient visit. The interviews were performed by the PEA at the practice sites and lasted approximately 30 to 40 minutes. Answers to questions were recorded as written notes and entered into a computerized database. Two of the study investigators (DCS, KR), masked to PCP and practice identities, independently classified practice activities for each CRCS step into general modalities or acting personnel) then subsequently reviewed them together and reached a consensus about differences. One of the investigators (DCS) subsequently repeated the structured interview by telephone with each PCP who was classified as an exemplar (higher rates of CRCS; see Analysis, below).

\section{Medical Record Abstractions}

PEAs reviewed the medical records of patients eligible for CRCS for each PCP to determine performance of CRCS that met guidelines ${ }^{1-5}$ at the time of the survey. Patients were eligible for CRCS if they (1) were $\geq 51$ and $<80$ years old, (2) had been in the practice for at least 12 months before the audit, and (3) had been seen for an office visit at least once during the previous year. Patients with previous colorectal cancer or who were evaluated because of symptoms were excluded. CRCS history up to the preceding 10 years was abstracted from the medical record. Any documentation of shortterm outcomes (eg, adequate examination, findings, and complications) for each CRCS test was recorded. Additional information about each patient, including comorbidities and adherence to other preventive services, was recorded. Chart abstraction reliability and validity for each PEA were checked by comparison with 2 of the study investigators (DCS, KR).

\section{Analysis}

Using methods similar to previous OKPRN studies, ${ }^{18,26}$ we initially defined "exemplars" using the benchmark performance of the 75 th percentile or higher for any recommended CRCS method, ${ }^{4}$ which in this sample was $52 \%$. During the medical records abstraction process and observation of practice procedures, it became obvious that 3 PCPs who provided endoscopy (colonoscopy $[\mathrm{n}=2]$ and flexible sigmoidoscopy $[\mathrm{n}=1]$ ) had very high rates of performing CRCS methods but frequently elicited symptoms from patients before they underwent endoscopy and subsequently coded a diagnosis other than screening, which excluded them from the analysis (ie, symptomatic, not eligible for screening). Although we did not change the medical record exclusion criteria and abstracted only the medical records of patients who were eligible for screening, because it seemed inappropriate to classify these PCPs as nonexemplars, we lowered the criterion for screening performance to $47 \%$ to include them as exemplars. Thus, the total number of exemplars was 16. The exemplars were drawn from 10 practices: academic faculty $(\mathrm{n}=3)$, Indian Health Service $(\mathrm{n}=1)$, and private $(\mathrm{n}=6)$.

CRCS performance was measured by PCP, practice, and CRCS strategy. Patient characteristics such as sex, race, payor, medical problems, and provision of other preventive services were compared between exemplars and nonexemplars using the Pearson $\chi^{2}$ test. Continuous variables were compared between exemplars and nonexemplars using the independent Student $t$ test. Likert-scaled responses were compared using and independent samples median test and the Mann-Whitney $U$ test. CRCS recommendation and completion rates were compared using the Pearson $\chi^{2}$ test. Multivariate logistic regression modeling was used to determine the relationship between the PCP, patient, practice characteristics, and screening performance measures. We performed an exploratory analysis at the patient level to account for the clustering effects of PCP and practice. Because there were half as many sites as PCPs and there was heterogeneity among PCPs at the same site, only the 2-level analysis is presented. The $\alpha$ significance level for all analyses was set at $<.05$. Analyses were conducted using SPSS Statistics version 18.0 (IBM/SPSS, Inc., Chicago, IL).

\section{Results}

We abstracted 3596 medical records of 48 PCPs in 25 practices (mean, 75 records/PCP). All PCPs were family physicians (male, $\mathrm{n}=42$; female, $\mathrm{n}=$ 6). Although some PCPs were in academic practices, none were resident physicians. Patients of exemplars were slightly older (64.0 vs 63.2 years; 
$P=.015)$ and more of their patients were white than nonwhite $(50.8 \%$ vs $39.0 \% ; P<.001)$ (Table 1$)$. Patients of exemplars were more likely to have diagnoses of cancer $(4.3 \%$ vs $3.0 \% ; P=.029)$ or renal failure $(2.4 \%$ vs $1.1 \% ; P=.006)$. They more often received other preventive services, such as cholesterol screening $(90.3 \%$ vs $83.9 \% ; P<.001)$ and mammograms (66.9\% vs $49.4 \%$; $P<.001)$. Patients who were white and insured were more likely to be screened, and exemplars had more of these patients; however, exemplars performed CRCS better than nonexemplars for patients who were nonwhite $(49.2 \%$ vs $24 \%$; $P<.001)$ and uninsured $(38.5 \%$ vs $12.8 \% ; P<.001)$. PCP variables (age, years since graduation, rural/ urban, or academic/nonacademic) were not significantly associated with CRCS.

Documentation of a screening recommendation was higher for exemplars within the past 1 - and 5 -year time periods (Table 2). Overall, rates of CRCS were higher for exemplars $(57.2 \%$ vs $27.6 \%$; $P<.001)$. Patients of exemplars had significantly higher rates of screening with fecal occult blood test (FOBT), colonoscopy, and flexible sigmoidoscopy but not double-contrast barium enemas. Colonoscopy rates also were higher for exemplars, excluding endoscopists (43.4\% vs $17.8 \% ; P<.001$ ).

Medical records from exemplars contained a tracking method more frequently than nonexem-

Table 1. Characteristics of Patients of Exemplars and Nonexemplars

\begin{tabular}{|c|c|c|c|c|c|}
\hline \multirow[b]{2}{*}{ Patient Characteristics } & \multicolumn{2}{|c|}{ Exemplars ( $\mathrm{n}=1188)$} & \multicolumn{2}{|c|}{$\begin{array}{l}\text { Non-exemplars } \\
\quad(\mathrm{n}=2408)\end{array}$} & \multirow[b]{2}{*}{ Significance p-value } \\
\hline & No. & $\%$ & No. & $\%$ & \\
\hline \multicolumn{6}{|l|}{ Sex } \\
\hline Female & 738 & 62.3 & 1470 & 61.1 & NS \\
\hline Missing & 3 & 0.3 & 2 & 0.1 & NS \\
\hline Age (mean years) & 64.0 & 4.8 & 63.2 & 3.4 & .015 \\
\hline \multicolumn{6}{|l|}{ Race } \\
\hline White & 604 & 50.8 & 940 & 39.0 & $<.001$ \\
\hline Black & 173 & 14.6 & 244 & 10.1 & $<.001$ \\
\hline Native American & 116 & 9.8 & 171 & 7.1 & $<.007$ \\
\hline Asian Pacific & 10 & 0.8 & 177 & 7.4 & $<.001$ \\
\hline Missing & 285 & 24.0 & 876 & 36.4 & $<.001$ \\
\hline \multicolumn{6}{|l|}{ Payor } \\
\hline Commercial & 453 & 38.1 & 1009 & 41.9 & .03 \\
\hline Medicare & 453 & 38.1 & 928 & 38.5 & NS \\
\hline Medicaid & 91 & 7.7 & 141 & 5.9 & .04 \\
\hline Indian Health Service & 94 & 7.9 & 115 & 4.8 & $<.001$ \\
\hline Self-pay & 26 & 2.2 & 109 & 4.5 & $<.001$ \\
\hline Missing & 71 & 6.0 & 106 & 4.4 & NS \\
\hline \multicolumn{6}{|l|}{ Medical Problems } \\
\hline Cancer & 4.3 & 51 & 3.0 & 72 & .029 \\
\hline COPD & 9.3 & 110 & 10.8 & 259 & NS \\
\hline Dementia & 1.3 & 16 & 1.0 & 25 & NS \\
\hline Depression & 17.5 & 208 & 15.0 & 361 & NS \\
\hline Diabetes & 24.7 & 293 & 24.6 & 593 & NS \\
\hline Heart disease & 16.6 & 197 & 15.5 & 373 & NS \\
\hline Hypertension & 56.8 & 675 & 58.2 & 1402 & NS \\
\hline Renal failure & 2.4 & 28 & 1.1 & 27 & .006 \\
\hline Stroke & 2.4 & 29 & 2.3 & 55 & NS \\
\hline \multicolumn{6}{|l|}{ Preventive services* } \\
\hline Influenza vaccination & 364 & 45.3 & 772 & 42.1 & NS \\
\hline Cholesterol screening & 1066 & 90.3 & 1998 & 83.9 & $<.001$ \\
\hline Mammogram & 496 & 67.4 & 726 & 49.8 & $<.001$ \\
\hline
\end{tabular}

*Excludes services refused or not indicated.

COPD, chronic obstructive pulmonary disease; NS, not statistically significant. 
Table 2. Colorectal Cancer Screening Performance

\begin{tabular}{|c|c|c|c|c|c|c|c|}
\hline \multirow{2}{*}{$\frac{\text { Screening }}{\text { Recommended screening period (years) }}$} & \multicolumn{3}{|c|}{ Exemplar Patients $(\mathrm{n}=1188)$} & \multicolumn{3}{|c|}{$\begin{array}{l}\text { Nonexemplar Patients } \\
\quad(\mathrm{n}=2408)\end{array}$} & \multirow[b]{2}{*}{$P$} \\
\hline & $\%$ & No. & Total no.* & $\%$ & No. & Total no.* & \\
\hline 1 & 54.6 & 572 & 1047 & 32.7 & 732 & 2238 & $<.001$ \\
\hline 5 & 57.0 & 470 & 829 & 34.9 & 698 & 1998 & $<.001$ \\
\hline 10 & 23.8 & 72 & 302 & 18.9 & 184 & 972 & .063 \\
\hline \multicolumn{8}{|l|}{ Documented screening method } \\
\hline Fecal occult blood test & 205 & 17.3 & & 259 & 10.8 & & $<.001$ \\
\hline Colonoscopy & 456 & 38.4 & & 448 & 18.6 & & $<.001$ \\
\hline Flexible sigmoidoscopy & 29 & 2.4 & & 31 & 1.3 & & .018 \\
\hline Double-contrast barium enema & 24 & 2.0 & & 20 & 0.8 & & .003 \\
\hline Any screening method & 652 & 54.9 & & 682 & 28.3 & & $<.001$ \\
\hline
\end{tabular}

*Patients appropriate for screening during each screening period minus those missing.

plars, but it was used only slightly more often (Table 3). However, when the medical record did not contain a tracking method or the tracking method was not used, patients of exemplars had similar rates of CRCS that were consistently higher than those of the patients of nonexemplars.

Insurance coverage had a major effect on CRCS rates for both exemplars and nonexemplars (Table 4). Patients with Medicare or commercial insurance had higher screening rates than patients who were self-payers or covered by Medicaid or the Indian Health Service for both exemplars and nonexemplars. For all payor types, patients of exemplars had higher screening rates than those of nonexemplars. Self-paying patients of exemplars had a screening rate 3 times higher than those of nonexemplars (38.5\% vs $12.8 \% ; P=.004)$. Most exemplars recommended FOBT for self-paying patients. One exemplar negotiated a price reduction with a local hospital for colonoscopy for patients without insurance.

Exemplars did not use tracking systems to determine whether patients who agreed to endoscopies actually had endoscopies performed (unless the exemplar himself was an endoscopist). Instead they relied on questioning patients with no documentation of the endoscopy during a subsequent visit. One exemplar used a computerized tracking system that was limited to follow-up of positive FOBTs. Although the numbers of abnormal CRCS results were small, exemplars did not seem to have higher rates of follow-up for abnormal CRCS results than nonexemplars (Table 5).

In their questionnaire responses, fewer exemplars indicated that they were planning significant changes in the way they delivered CRCS services in their practice (exemplars, $0 \%$ vs nonexemplars, $22 \% ; P=.07)$. Exemplars estimated a much higher rate of newly diagnosed colorectal polyps in their practice than nonexemplars $(21.8 \%$ vs $7.6 \% ; P=$ $.074)$, and most polyps were detected by colonoscopy $(86.8 \%$ vs $52.1 \%$; $P<.001)$. Estimates of newly diagnosed colorectal cancers were not significantly different.

Because there were half as many sites as PCPs and there was heterogeneity among PCPs at the same site, only a 2-level multivariate analysis was performed. No variables describing PCPs (age,

Table 3. Rates of Colorectal Cancer Screening in Exemplars and Nonexemplars According to the Presence of Tracking Method

\begin{tabular}{|c|c|c|c|c|c|c|}
\hline \multicolumn{2}{|c|}{ Tracking Method } & \multicolumn{2}{|c|}{ Exemplar Patients $(\mathrm{n}=1188)$} & \multicolumn{2}{|c|}{ Non-exemplar Patients $(\mathrm{n}=2408)$} & \multirow[b]{2}{*}{$P$ value } \\
\hline Present & Used & Subtotal & Screened & Subtotal & Screened & \\
\hline Yes & Yes & $619(56.9)$ & $359(58.1)$ & $1211(52.2)$ & $338(27.9)$ & $<.001$ \\
\hline Yes & No & $226(20.8)$ & 97 (42.9) & $313(13.5)$ & $79(25.2)$ & $<.001$ \\
\hline No & - & $315(22.4)$ & $171(54.3)$ & $858(34.3)$ & $250(29.1)$ & $<.001$ \\
\hline
\end{tabular}

Data shown as n (\%). 
Table 4. Colorectal Cancer Screening Performance Depending on Insurance Coverage

\begin{tabular}{lcrrr}
\hline & \multicolumn{3}{c}{ Patients } & \\
\cline { 2 - 3 } Insurance Coverage & Screened (n) & Total (n) & $\%$ & $P$ value* \\
\hline $\begin{array}{l}\text { Nonexemplars } \\
\quad \text { Medicare or }\end{array}$ & 588 & 1937 & 30.4 & \\
$\quad$ commercial & & & & \\
$\quad \begin{array}{l}\text { Medicaid or IHS } \\
\text { Self-pay }\end{array}$ & 48 & 256 & 19.1 & $<.001$ \\
Exemplars & 14 & 109 & 12.8 & \\
$\quad \begin{array}{l}\text { Medicare or } \\
\quad \text { commercial }\end{array}$ & 536 & 906 & 59.2 & \\
$\quad \begin{array}{l}\text { Medicaid or IHS } \\
\text { Self-pay }\end{array}$ & 72 & 185 & 38.9 & $<.001$ \\
\hline
\end{tabular}

IHS, Indian Health Service.

${ }^{*} P$ values for exemplars versus nonexemplars; comparisons were (1) Medicare or commercial, $P<.001$; (2) Medicaid or IHS, $P<$ .001 ; (3) self-pay, $P=.004$; and (4) total, $P<.001$.

years since graduation, rural/urban, or academic/ nonacademic) were significantly associated with CRCS. At the patient level, 3 variables were found to be associated with CRCS status: age, renal failure, and insurance coverage. While exemplars had more patients who were white or had some type of insurance, they performed better than nonexemplars in each group at risk for lack of CRCS (Table 4).

\section{PCP Interviews}

Exemplars rarely used interventions proposed in published studies. ${ }^{9}$ However, there were some spe-
Table 6. How Exemplars Screen for Colorectal Cancer

- Begin with a strong commitment to preventive services delivery.

- Use any visit to recommend colorectal cancer screening.

- Use paper or electronic health record to prompt screening recommendation.

- Offer a brief personal recommendation bundled with other preventive health services.

- Recommend colonoscopy or fecal occult blood testing (if colonoscopy is declined or not affordable).

- Emphasize benefits of early detection and avoiding regret.

- Determine barriers and use anecdotes to overcome.

- If colorectal cancer screening is declined, demonstrate concern and repeat recommendation during the next visit.

- Off-load explaining the details of the screening process to office staff.

- Cultivate a relationship with endoscopist(s) who provide quality service and communication, and outsource responsibility for the details of endoscopy process.

cific attitudes and techniques that exemplars used that, after reflection, they thought explained their improved performance (Table 6). Exemplars were highly motivated to provide CRCS and preventive services in general. Some had a personal experience themselves or with a relative, friend, or patient that increased their resolve to prevent colorectal cancer. Others had a strong orientation toward preventive services that was well integrated into their concept of clinical practice but also considered CRCS to be a priority. Two exemplars performed colonoscopy on patients within their practice and one performed sigmoidoscopy on patients referred from another large group practice.

Table 5. Documentation of Follow-up of Abnormal Colorectal Cancer Screening Tests

\begin{tabular}{|c|c|c|c|c|c|c|}
\hline \multirow[b]{2}{*}{ Screening Method } & \multicolumn{3}{|c|}{ Exemplars } & \multicolumn{3}{|c|}{ Nonexemplars } \\
\hline & Met Criteria (n) & Total (n) & $\%$ & Met Criteria (n) & Total (n) & $\%$ \\
\hline \multicolumn{7}{|c|}{ Fecal occult blood test } \\
\hline Recommended & 10 & 10 & 100 & 16 & 18 & 89 \\
\hline Results* & 9 & 10 & 90 & 8 & 16 & 50 \\
\hline \multicolumn{7}{|c|}{ Flexible sigmoidoscopy } \\
\hline Recommendation & 8 & 9 & 89 & 4 & 9 & 44 \\
\hline Results* & 3 & 7 & 43 & 3 & 9 & 33 \\
\hline \multicolumn{7}{|c|}{ Double-contrast barium enema } \\
\hline Recommendation & 5 & 7 & 71 & 2 & 6 & 33 \\
\hline Results* & 5 & 7 & 71 & 2 & 6 & 33 \\
\hline \multicolumn{7}{|l|}{ Colonoscopy } \\
\hline Recommendation & 181 & 231 & 78 & 176 & 218 & 81 \\
\hline Results* & 54 & 155 & 35 & 75 & 167 & 45 \\
\hline
\end{tabular}

*Patients were excluded from analysis if there was inadequate time for follow-up. 
Because of perceived competing demands, exemplars focused screening efforts on a limited number of conditions, typically breast cancer, cervical cancer, prostate cancer, hypercholesterolemia, and colon cancer $(\mathrm{n}=11)$. They usually initiated discussions with all patients around 50 years of age. For instance, one exemplar introduced these preventive services as "getting ready for the second half." Only 3 exemplars used a preventive visit. All exemplars reviewed either paper-based or computerized flow sheets at the time of the visit as a prompt strategy. Exemplars who used paper-based medical records said that it was critical that the flowsheet be in plain view when they opened the record. No exemplar used an automatic prompt or reminder system.

Exemplars used brief CRCS promotion scripts (labeled by one exemplar as a "spiel") that they could recite by memory during the interview. These scripts typically began with a preamble that introduced a rationale for CRCS based on a recommendation by an authority, eg, USPSTF recommendation $(\mathrm{n}=3)$; addressing the natural history of colorectal cancer and how CRCS prevents it ( $\mathrm{n}=3$ ); or as one of a list of recommendations endorsed by the PCP $(n=8)$. They did not focus on the risk of colorectal cancer. Instead they emphasized the benefits of early detection ("simple" removal of precancerous polyps during CRCS) and avoidance of regret ("It is easier to chop down a bush than a tree").

Exemplars did not present all CRCS methods to patients. Most mentioned colonoscopy first and no other method if colonoscopy was accepted $(\mathrm{n}=12)$. Three exemplars initially compared colonoscopy to FOBT. FOBT was recommended for patients who could not afford or did not want colonoscopy. Colonoscopy was presented as the most effective option, and the benefits of the 10-year follow-up period were emphasized. Barriers to colonoscopy, such as discomfort, were minimized by emphasizing sedation and analgesia $(n=11)$. Fear of discomfort was deflected by remarking that the bowel preparation was worse than the procedure $(n=7)$. Several exemplars noted that if patients expressed hesitation regarding colonoscopy they would switch to a discussion of FOBTs. Sigmoidoscopy and double-contrast barium enema were rarely mentioned or performed.

Exemplars used communication based on decision stage during CRCS discussions. For patients who were perceived to be in a precontemplative stage of change, exemplars relied on demonstrated concern and repetition of a consistent message $(\mathrm{n}=$ 15). For instance, if the patient was not ready for screening, one exemplar described how they altered the script to "[plant] the seed" for future discussions. Nearly all exemplars did not directly respond to resistance but would tell patients to talk to others, think about it, and they would discuss it again during the next visit. One exemplar handwrote a prescription for colonoscopy for patients "to fill" if they changed their minds. Exemplars estimated that patients initially in the precontemplative or contemplative stage of change needed to hear their CRCS recommendation 2 or 3 times before accepting screening.

Exemplars off-loaded the detailed description of the procedure, complications, cost, insurance coverage, and bowel preparation. If patients chose FOBT, this information was provided by ancillary office staff. Three of 4 exemplars that emphasized FOBT used the InSure fecal immunochemical test because it was considered easier for the patient to use, had increased accuracy, did not incur any costs to the practice, and was mailed directly to the laboratory for processing.

Six exemplars developed a relationship with one local endoscopist (or one who would travel to a local site), who would see the patient and perform the colonoscopy during one visit then promptly send a letter to both the PCP and the patient describing the results or would notify the PCP of the patient's failure to keep the appointment. The endoscopist was responsible for insurance authorization, scheduling, bowel preparation, counseling before and after the procedure counseling, and follow-up. All exemplars except one expressed the opinion that flexible sigmoidoscopy was not an economically viable screening option.

\section{Discussion}

There were 2 unexpected findings in our investigation of these CRCS practices. First, exemplars adopted few innovations proposed by researchers to improve CRCS to a greater extent than nonexemplars. ${ }^{9}$ While Patel et $\mathrm{al}^{7}$ found that visits for physicals had the strongest association with discussion of CRCS, exemplars favored opportunistic screening over preventive care visits. This may change with the advent of Medicare's "Welcome to Medi- 
care" and annual wellness visits. Exemplars seemed to have "programmed" themselves until it was habit to discuss CRCS bundled with a limited number of other preventive services with all patients more than 50 years old. This may have reduced the effect of patient-level barriers to recommendations for CRCS, such as comorbidities, failure to adhere to screening, distrust of medical interventions, or lack of insurance. ${ }^{27}$ While some studies have demonstrated the importance of assistance with scheduling endoscopy, ${ }^{28}$ exemplars approached this issue by developing clearly defined referral relationships with specific endoscopists who provided complete services during one face-to-face encounter that reduced the PCP's responsibility for scheduling, preparation, and follow-up of results.

The second unexpected finding was the relative importance of the use by exemplars of brief but sophisticated CRCS promotion scripts that informally paralleled theory-driven counseling techniques, such as communication based on decision stage and principles of motivational interviewing, during the patient visit. That experienced physicians' knowledge is organized to support flexible responses to variants in a familiar situation has been recognized in the theory of illness scripts, ${ }^{29,30}$ and physicians' scripts for procedures have been elicited by asking surgeons how they would handle patients who are briefly described to them. ${ }^{31}$ However, this is the first study we know of in which PCPs have reported their own scripts. The emphasis on colonoscopy in CRCS scripts used by exemplars is congruent with the extended parallel process model in which fear is aroused by increasing the perceived threat and then making recommendations of highefficacy responses to manage that fear effectively. ${ }^{32}$ In the case of colonoscopy, the fear of colorectal cancer was aroused by PCPs and then mitigated by the offer of an intervention that removes polyps, thereby reducing the threat of colorectal cancer. It is important to note that exemplars did not use numerical expressions of colorectal cancer risk, nor did they attempt to generate high levels of fear. Instead they motivated patients by priming feelings of anticipated regret.

Exemplars also relied on motivational interviewing techniques to improve adherence to CRCS. ${ }^{33}$ When patients were ambivalent, PCPs explored the causes of the ambivalence and attempted to address them, frequently with an anecdote. They "rolled with resistance," avoiding direct arguments, and were respectful and supportive of patient autonomy. Exemplars leveraged the rapport developed during their longitudinal relationship with the patient over time to promote screening while sensitively gauging the intensity of their message to the patient's stage of change, being cognizant of the power of a consistent message over time.

PCPs' scripts generally precluded discussion of patient preferences and choice of CRCS test. This is similar to the findings of McQueen et $\mathrm{al}^{13}$ in which analysis of audio-recorded visits revealed that physicians consistently recommended colonoscopy to the exclusion of other tests. However, in this study, although exemplars focused on colonoscopy, they reported that they resorted to FOBT when required by a patient's rejection of the initial recommendation or financial circumstances. A more complete shared decision-making approach, presenting the pros and cons of all screening methods, as recommended by the USPSTF, was not used by any exemplars. $^{2}$ In at least 2 studies, patients who were offered a choice of CRCS modalities were less likely to be screened. ${ }^{28}$ It has been theorized that as the number of alternatives increases, individuals may perceive information overload, ${ }^{14,34}$ although incomplete information may also hinder decision making. ${ }^{35}$

Despite the lack of quality evidence supporting effectiveness, ${ }^{36}$ exemplars considered colonoscopy superior to other screening tests. This is consistent with the findings of Zapka et $\mathrm{al},{ }^{37}$ who reported that $86 \%$ of surveyed PCPs strongly agreed that colonoscopy is the best CRCS test. Zapka et al also reported that $58 \%$ of the respondents were worried they could be sued if they did not recommend colonoscopy. We did not ask this question in our study, but fear of lawsuits was not mentioned in any interview.

Zapka et $\mathrm{al}^{37}$ reported that $53 \%$ of PCPs had decreased their volumes of flexible sigmoidoscopy over the past 3 years but did not offer an explanation. Nearly all PCPs in our study did not consider flexible sigmoidoscopy a viable economic alternative for their practice. If flexible sigmoidoscopy is to remain a alternative CRCS method, issues of perceived inferiority and economic incentives will need to be addressed. ${ }^{36}$ Likewise, the clinic costs of CRCS with the FOBT process may be a disincentive to screening. Several exemplars outsourced preparation of FOBT kits and processing of FOBT specimens to national laboratories, who then reported results. While focusing on colonoscopy to 
the exclusion of other CRCS methods has been associated with decreased adherence rates, ${ }^{38}$ exemplars in this study used a flexible approach, promoting FOBT as an alternative.

The major strength of this study is the linkage between CRCS performance and in-depth exploration of PCP health promotion behaviors. By having an objective measure of CRCS effectiveness, PCP counseling techniques in particular could be validated. There have been numerous large-scale surveys of PCPs' CRCS recommendations and practices, but these were rarely coupled with performance. ${ }^{39} \mathrm{Al}-$ though there have been a large number of clinical trials that tested various CRCS interventions in primary care settings, this is one of a few studies to describe methods adopted by PCPs and determine their relationship to the performance of CRCS.

There are some limitations to this study. The cross-sectional design does not allow causal conclusions, and there may be unmeasured confounding variables. Relatively few PCPs were studied, and all were from Oklahoma. However, there was a range of ages and rural, suburban, and urban practice settings represented among PCPs. While both exemplars and nonexemplars completed an in-depth, face-to-face structured interview, only exemplars had follow-up telephone interviews, limiting direct comparison with nonexemplars. In addition, the techniques described by exemplars suffer from a lack of objective verification. It may be that it is not the techniques they use but their competence using them that differentiates their performance of CRCS. At first glance it may seem that exemplars did not achieve notably high rates of CRCS (52\%) compared with the national average. However, the exclusion criteria for the medical record abstractions in this study were extremely stringent so that patients who had ever had symptoms suggestive of colorectal cancer in the past (eg, constipation) or were previously found to have colon polyps were excluded. Use of CRCS tests nationally in the Behavioral Risk Factor Surveillance System surveys are self-reported and do not exclude symptomatic patients who may have had tests. ${ }^{6}$

\section{Conclusion}

We describe the use of brief but sophisticated CRCS promotion scripts ("spiels"), which informally paralleled theory-driven counseling techniques, by motivated, experienced PCPs. Future research should be directed toward whether these techniques can be used to create an intervention aimed at physicians to improve CRCS. Colonoscopy was the preferred screening method, and FOBT was recommended for patients who could not afford or did not prefer colonoscopy. Flexible sigmoidoscopy or barium enemas were rarely recommended. There was minimal use of system interventions to improve CRCS that were often proposed by researchers, and more consideration was given to the efficiency and lower office costs of providing preventive services.

\section{References}

1. Levin B, Lieberman DA, McFarland B, et al. Screening and surveillance for the early detection of colorectal cancer and adenomatous polyps, 2008: a joint guideline from the American Cancer Society, the US Multi-Society Task Force on Colorectal Cancer, and the American College of Radiology. Gastroenterology 2008;134:1570-95.

2. U.S. Preventive Services Task Force. Screening for colorectal cancer: U.S. Preventive Services Task Force recommendation statement. Ann Intern Med 2008;149:627-37.

3. Yee J, Rosen MP, Blake MA, et al. ACR Appropriateness Criteria on colorectal cancer screening. J Am Coll Radiol 2010;7:670-8.

4. Rex DK, Johnson DA, Anderson JC, Schoenfeld PS, Burke CA, Inadomi JM; American College of Gastroenterology. American College of Gastroenterology guidelines for colorectal cancer screening 2009 [corrected]. Am J Gastroenterol 2009;104:739-50.

5. Qaseem A, Denberg TD, Hopkins RH Jr, et al. Screening for colorectal cancer: a guidance statement from the American College of Physicians. Ann Intern Med 2012;156:378-86.

6. Centers for Disease Control and Prevention (CDC). Use of colorectal cancer tests-United States, 2002, 2004, and 2006. MMWR Morbid Mortal Wkly Rep 2008;57:253-8.

7. Patel P, Forjuoh SN, Avots-Avotins A, Patel T. Identifying opportunities for improved colorectal cancer screening in primary care. Prev Med 2004;39:239-46.

8. Taylor V, Lessler D, Mertens K, et al. Colorectal cancer screening among African Americans: the importance of physician recommendation. J Natl Med Assoc 2003;95:806-12.

9. Holden DJ, Jonas DE, Porterfield DS, Reuland D, Harris R. Systematic review: enhancing the use and quality of colorectal cancer screening. Ann Intern Med 2010;152:668-76.

10. Taylor ML, Anderson R. Colorectal cancer screening: physician attitudes and practices. WMJ 2002; 101:39-43.

11. Klabunde CN, Lanier D, Nadel MR, McLeod C, Yuan G, Vernon SW. Colorectal cancer screening 
by primary care physicians: recommendations and practices, 2006-2007. Am J Prev Med 2009;37:8-16.

12. Nemeth LS, Nietert PJ, Ornstein SM. High performance in screening for colorectal cancer: a Practice Partner Research Network (PPRNet) case study. J Am Board Fam Med 2009;22:141-6.

13. McQueen A, Bartholomew LK, Greisinger AJ, et al. Behind closed doors: physician-patient discussions about colorectal cancer screening. J Gen Intern Med 2009;24:1228-35.

14. Ling BS, Trauth JM, Fine MJ, et al. Informed decision-making and colorectal cancer screening: is it occurring in primary care? Med Care 2008;46(9 Suppl 1):S23-9.

15. Lafata JE, Cooper GS, Divine G, et al. Patientphysician colorectal cancer screening discussions: delivery of the 5A's in practice. Am J Prev Med 2011;41:480-6.

16. Ferrell CW, Mold JW, Aspy CB. Management of prescription refills in primary care: an Oklahoma Physicians Resource/Research Network (OKPRN) study. J Am Board Fam Med 2006;19:31-8.

17. Mold JW, Cacy DS, Dalbir DK. Management of laboratory test results in family practice. An OKPRN study. Oklahoma Physicans Resource/Research Network. J Fam Pract 2000;49:709-15.

18. Mold JW, Gregory ME. Best practices research. Fam Med 2003;35:131-4.

19. Mold JW, Mathew MK, Belgore S, DeHaven M. Prevalence of night sweats in primary care patients: an OKPRN and TAFP-Net collaborative study. J Fam Pract 2002;51:452-6.

20. Nagykaldi Z, Mold JW. Diabetes Patient Tracker, a personal digital assistant-based diabetes management system for primary care practices in Oklahoma. Diabetes Technol Ther 2003;5:997-1001.

21. Lemelin J, Hogg W, Baskerville N. Evidence to action: a tailored multifaceted approach to changing family physician practice patterns and improving preventive care. CMAJ 2001;164:757-63.

22. DOPC Writing Group. Conducting the Direct Observation of Primary Care Study. J Fam Pract 2001; 50:345-52.

23. Cabana MD, Rand CS, Powe NR, et al. Why don't physicians follow clinical practice guidelines? A framework for improvement. JAMA 1999;282: $1458-65$.

24. Woolf SH. Overcoming the barriers to change: screening for colorectal cancer. Am Fam Physician 2000;61:1621-2, 1628 .
25. Pommerenke FA, Dietrich A. Improving and maintaining preventive services. Part 1: applying the patient path model. J Fam Pract 1992;34:86-91.

26. Johnson BJ, Mold JW, Pontious JM. Reduction and management of no-shows by family medicine residency practice exemplars. Ann Fam Med 2007;5:534-9.

27. Guerra CE, Schwartz JS, Armstrong K, Brown JS, Halbert CH, Shea JA. Barriers of and facilitators to physician recommendation of colorectal cancer screening. J Gen Intern Med 2007;22:1681-8.

28. Lafata JE, Divine G, Moon C, Williams LK. Patientphysician colorectal cancer screening discussions and screening use. Am J Prev Med 2006;31:202-9.

29. Charlin B, van der Vleuten C. Standardized assessment of reasoning in contexts of uncertainty: the script concordance approach. Eval Health Prof 2004; 27:304-19.

30. Schmidt HG, Norman GR, Boshuizen HP. A cognitive perspective on medical expertise: theory and implication. Acad Med 1990;65:611-21.

31. Abernathy CM, Hamm RM. Surgical scripts: master surgeons think aloud about 43 common surgical problems. Philadelphia, PA: Hanley \& Belfus; 1994.

32. Pengchit W, Walters ST, Simmons RG, et al. Motivation-based intervention to promote colonoscopy screening: an integration of a fear management model and motivational interviewing. J Health Psychol 2011;16:1187-97.

33. Wahab S, Menon U, Szalacha L. Motivational interviewing and colorectal cancer screening: a peek from the inside out. Patient Educ Couns 2008;72: $210-7$.

34. Iyengar SS, Lepper MR. When choice is demotivating: can one desire too much of a good thing? J Pers Soc Psychol 2000;79:995-1006.

35. Hoffman RM, Lewis CL, Pignone MP, et al. Decision-making processes for breast, colorectal, and prostate cancer screening: the DECISIONS survey. Med Decis Making 2010;30(5 Suppl):53S-64S.

36. Inadomi JM. Why you should care about screening flexible sigmoidoscopy. N Engl J Med 2012;366: 2421-2.

37. Zapka JM, Klabunde CN, Arora NK, Yuan G, Smith JL, Kobrin SC. Physicians' colorectal cancer screening discussion and recommendation patterns. Cancer Epidemiol Biomarkers Prev 2011;20:509-21.

38. Inadomi JM, Vijan S, Janz NK, et al. Adherence to colorectal cancer screening: a randomized clinical trial of competing strategies. Arch Intern Med 2012; 172:575-82.

39. Klabunde CN, Frame PS, Meadow A, Jones E, Nadel $\mathrm{M}$, Vernon SW. A national survey of primary care physicians' colorectal cancer screening recommendations and practices. Prev Med 2003;36:352-62. 